ISSN: 0514-7336

DOI: http://dx.doi.org/10.14201/zephyrus2015757384

\title{
LAS REPRESENTACIONES LEVANTINAS DE BOVINOS DE LA CASA FORESTAL DE TORMÓN (TERUEL): CEJA DE PIEZARRODILLA Y CERRADA DEL TÍO JORGE
}

\section{Levantine bovine representations from Casa Forestal de Tormón (Teruel): Ceja de Piezarrodilla and Cerrada del Tío Jorge}

\author{
Manuel Bea Martínez* y Jorge Angás Pajas** \\ * Dpto. de Ciencias de la Antigüedad. Facultad de Filosofía y Letras. Cl Pedro Cerbuna, 12. 50009 Zaragoza. \\ Correo-e:manubea@unizar.es \\ ** Scanner Patrimonio e Industria. Avda. Navarra, 103 (local). 50017 Zaragoza. Correo-e: j.angas@3dscanner.es
}

Recepción: 3/01/2015; Revisión: 6/02/2015; Aceptación: 25/03/2015

BIBLID [0514-7336 (2015) LXXV, enero-junio; 73-84]

Resumen: Se presenta el análisis, mediante nuevas técnicas de documentación, de dos de los conjuntos rupestres con figuras de bóvidos levantinas más destacadas de la Sierra de Albarracín: Ceja de Piezarrodilla y Cerrada del Tío Jorge. Pasados más de 30 años desde el último estudio exhaustivo realizado sobre estos conjuntos, el tratamiento digital de la imagen nos ha permitido no sólo documentar mejor las representaciones, sino ofrecer una nueva lectura relativa a la conservación de las pinturas en determinados motivos, apuntando al crecimiento de agentes bióticos sobre los pigmentos del toro de Ceja de Piezarrodilla.

El reestudio de los conjuntos rupestres de la zona, junto al realizado sobre nuevos abrigos recientemente descubiertos, ha permitido establecer paralelos temáticos y estilísticos que subrayan la conformación en la Sierra de Albarracín de un verdadero núcleo rupestre con particularidades que poco tienen que ver con el arte levantino propio de otras áreas geográficas.

Palabras clave: Sierra de Albarracín; arte levantino; bóvidos; calcos digitales; estilo; conservación.

AвSTRACT: A new analysis on two of the most interesting rock-art shelters of the mountain range of Albarracín is presented. New documentation techniques have been used on the study. It has been more than 30 years since the last exhaustive study carried on these sites, the digital enhancement of the images has allow us to have not only a better documentation of the motifs but also offer a new reading relating to the preservation of the paintings. In this way, we point out that one the re-painted of the bull from Ceja de Piezarrodilla could be, in fact, a biotic agent growing over the painting.

The re-study of the rock-art sites of the area, also with the new rock-art shelters recently found, allow us to establish thematic and stylistic parallels that highlight the formation of a real rock-art nucleus in the mountain range of Albarracín with some particularities that have little to do with the Levantine rock-art in other areas.

Key words: Mountain range of Albarracín; Levantine rock-art; bovid; digital tracing; style; conservation. 


\section{Introducción}

El presente trabajo se enmarca en dos campos de actuaciones bien definidos ${ }^{1}$ en los que se ha contemplado la realización de una documentación integral de los conjuntos rupestres así como el estudio estilístico y compositivo de los mismos.

Tradicionalmente los abrigos rupestres de la Sierra de Albarracín han ocupado un puesto de privilegio dentro de los estudios sobre arte levantino debido, en gran medida, a la excepcionalidad de las representaciones de grandes bóvidos en blanco y en negro, algunos de éstos repintados, estableciéndose su importancia al definirse como propios de las primeras fases de desarrollo del arte levantino (Beltrán, 1968; Ripoll, 1968; Piñón, 1982) o, más recientemente, por haberse redefinido algunas de las bases cronoculturales de determinados conjuntos (Cruz, 2004 y 2005; Martínez Bea, 2005 y 2008). En todo caso, y con respecto a aspectos estilísticos y temáticos, las características propias del arte rupestre de la Sierra de Albarracín lo definen como una verdadera excepcionalidad, introduciendo ..."elementos tan poco de acuerdo con lo conocido que obligarán a un planteamiento de este foco regional de la expresión gráfica parietal”... (Beltrán, 1995: 314).

El presente estudio nace de la necesidad de realizar un análisis actualizado de los conjuntos rupestres de la zona, la más meridional del núcleo de la Sierra de Albarracín, siendo el análisis exhaustivo más reciente publicado el de F. Piñón, hace más de 30 años (Piñón, 1982). En esta línea, son precisamente las grandes representaciones de bovinos uno de los elementos temáticos y estilísticos más interesantes, destacados y definitorios. El proyecto de estudio integral de los conjuntos rupestres ha contemplado la documentación geométrica de los mismos mediante escáner láser de los abrigos así como el uso de escáner de luz blanca estructurada para la zona concreta de los paneles decorados. Asimismo, se han realizado calcos digitales de cada uno de los

\footnotetext{
1 Trabajo vinculado a los proyectos "Estudio integral de las pinturas rupestres del núcleo de Tormón y del Barranco de las Olivanas", financiado por la Dirección General de Bellas Artes y Bienes Culturales (Ministerio de Educación, Cultura y Deporte) y “Transiciones climáticas y adaptaciones sociales en la Prehistoria de la cuenca del Ebro" (HAR2014-59042-P), así como al Grupo "Primeros Pobladores del Valle del Ebro" (H-07).
}

motivos a partir de fotografías calibradas con carta colorimétrica y tratamiento digital de imagen mediante DStretch@.

Las líneas que siguen se centran en el análisis de los dos conjuntos clásicos con figuraciones de grandes toros de la zona de Tormón: Cerrada del Tío Jorge y Ceja de Piezarrodilla y de su relación temático-estilística con otros paralelos levantinos de la zona y áreas geográficas próximas.

\section{Descripción de los conjuntos decorados}

Los conjuntos con representaciones de bóvidos objeto de estudio se localizan a unos $25 \mathrm{~km}$ al s de Albarracín (Fig. 1), a unos $7 \mathrm{~km}$ al NE del término municipal de Tormón, en una zona de pinar en la que se abre el denominado Prado de Tormón, espacio abierto de grandes dimensiones y de carácter estratégico al converger en él diversos barrancos.

\subsection{Cerrada del Tío Jorge}

Este conjunto recibe el nombre de "la cerrada" o corral que se adosaba al abrigo decorado ${ }^{2}$. Se trata de un abrigo de dimensiones medias ( $3 \mathrm{~m}$ de altura y $2 \mathrm{~m}$ de profundidad máxima) y situado en el mismo "Prado de Tormón", a unos $150 \mathrm{~m}$ al o de la Casa Forestal, en la base de una formación arenisca de grandes dimensiones.

Aunque el abrigo fue descubierto por $\mathrm{H}$. Breuil en 1924, el primer y escueto acercamiento a las pinturas contenidas en el conjunto fue el realizado por H. Obermaier (1927), siendo objeto, al igual que ocurriría con el abrigo de los Toros de las Olivanas, de un estudio más detallado por parte del investigador hispano-alemán junto al propio Breuil

\footnotetext{
2 Cabe señalar la doble nomenclatura existente para este conjunto que es referido en los primeros trabajos como "Cerrada del Tío José" (Obermaier y Breuil, 1927: 530; Hernández-Pacheco, 1959: 413; Beltrán, 1968: 147 y 1993: 159) mientras que en otros estudios es referido como "Cerrada del Tío Jorge" (Almagro, 1970: 360; Piñón, 1982: 144; Collado, 1992: 21; Utrilla, 2000: 54), llegándose a emplear ambas toponimias de forma indistinta (Beltrán, 1986: 44). En el presente estudio hemos adoptado la toponimia oficial aparecida en el inventario de arte rupestre de la Dirección General de Patrimonio Cultural del Gobierno de Aragón.
} 
(Obermaier y Breuil, 1927). Posteriores trabajos de síntesis (Hernández-Pacheco, 1959: 413; Beltrán, 1968: 147; Almagro, 1970: 360) recogieron la pintura contenida en este abrigo, si bien el trabajo exhaustivo más reciente, hasta el presente, se correspondía con el realizado por Piñón (1982).

A poco menos de $2 \mathrm{~m}$ con respecto al suelo natural, se pintó la figura de un bóvido en color rojo castańo oscuro orientado a la izquierda. Tan sólo se conserva la mitad anterior del animal, con parte de las patas y alcanzando $43,5 \mathrm{~cm}$ de longitud máxima. El motivo completo debería contar con unas dimensiones notables, aspecto recurrente en otras figuraciones de toros de la Sierra de Albarracín.

El estilo con el que se realizó la figura concuerda con el observado en otras representaciones de bóvidos de la zona: una tendencia naturalista en la que algunos elementos corporales resultan ligeramente desproporcionados, en este caso la cabeza aparece reducida respecto a la potencia con la que fue realizado el cuello del animal. Los cuernos, relativamente finos, fueron llevados a cabo de perfil con la típica morfología de medialuna. El bóvido aparece con el cuello inclinado captando un momento de gran tensión, lo que podría inducir a interpretar su actitud de embestida.

De los estudios previos realizados sobre el conjunto,
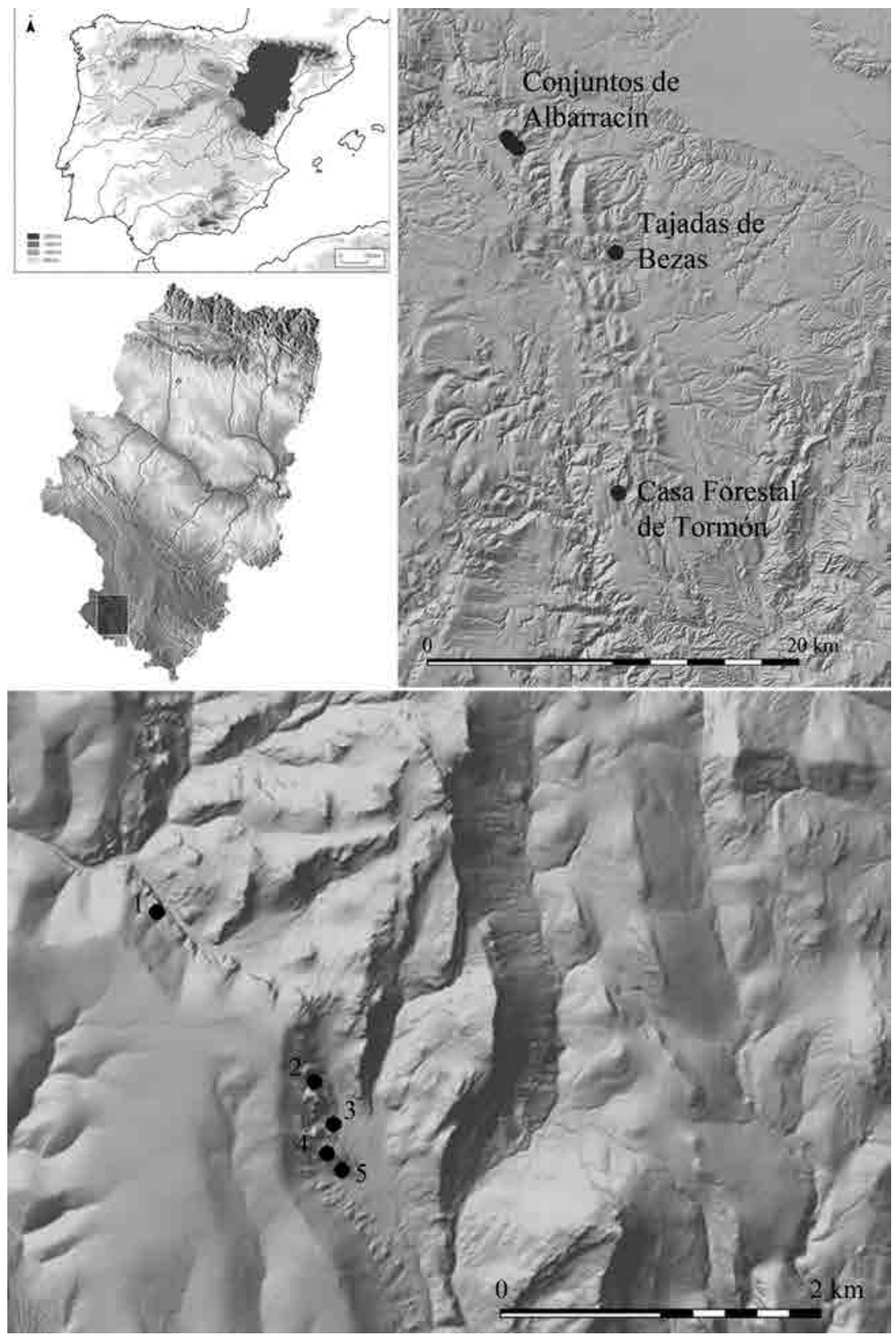

FIg. 1. Arriba: Localización de los conjuntos rupestres de la Casa Forestal de Tormón en el contexto del arte rupestre de la Sierra de Albarracín. Abajo: conjuntos rupestres de los alrededores de la Casa Forestal de Tormón: 1) Prado de las Olivanas; 2) Ceja de Piezarrodilla; 3) Cerrada del Tio Jorge; 4) Cabras Blancas; 5) Paridera de Tormón. existe una marcada diferenciación en cuanto a la definición de la propia figura, evidente en la plasmación de los calcos resultantes. Así, en el ofrecido por Obermaier y Breuil se dice que ..."una línea delgada y

derecha parece representar una azagaya clavada en el pecho"... (Obermaier y Breuil, 1927: 530-531). Este interesante elemento no aparece referido en el estudio realizado por Piñón (1982: 146-147, 

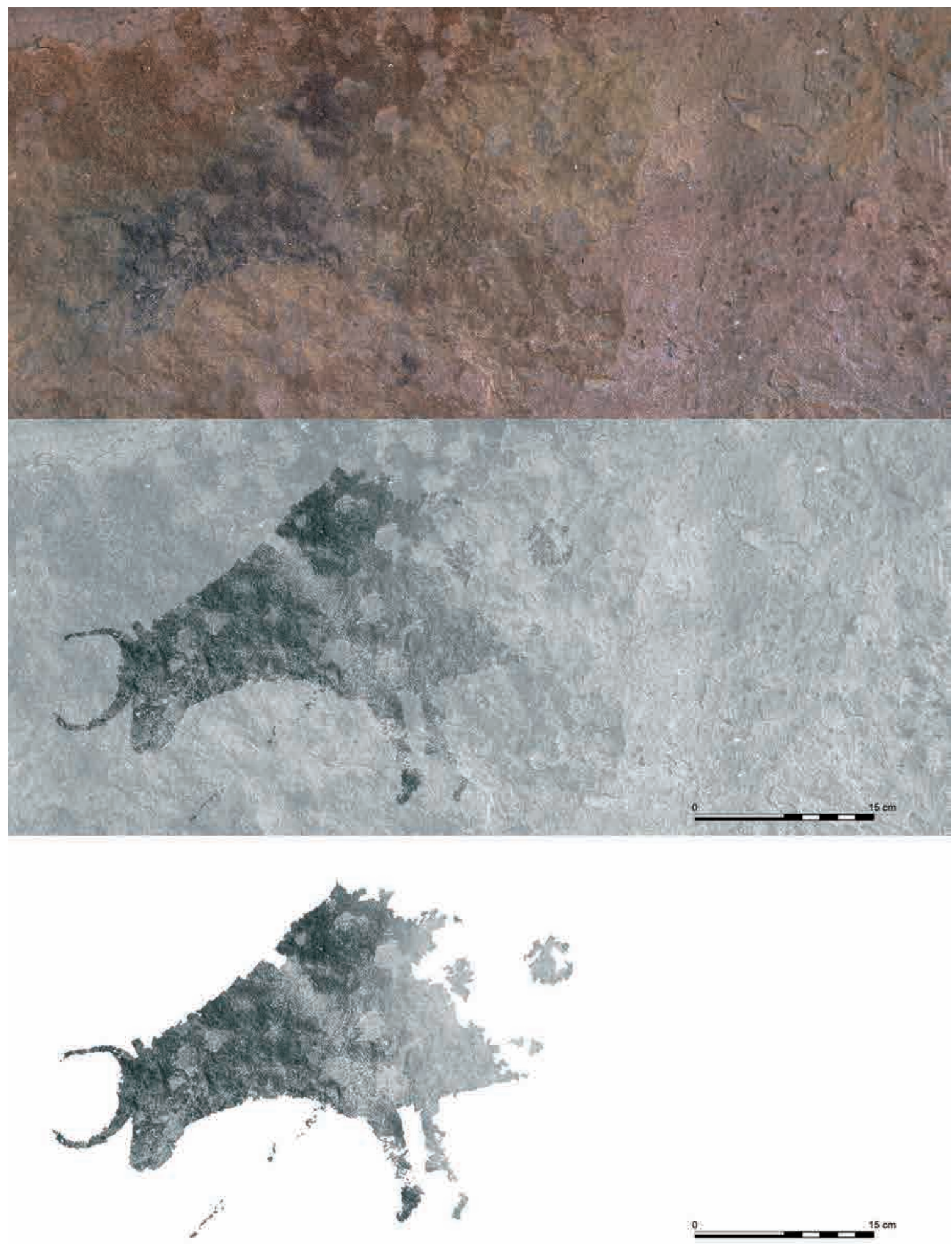

Fig. 2. Fotografía y calcos del toro de la Cerrada del Tío Jorge. 
fig. 37), en el que tan sólo se describe el tercio delantero del animal, cuello y cabeza con parte inicial del pecho y de la cruz del mismo (Fig. 2, n.o 2). Tampoco se hacen eco de la condición de animal herido trabajos posteriores (Beltrán, 1986), a pesar del evidente interés interpretativo y de la descripción del animal como "derrotado" en alguno de esos trabajos (Beltrán, 1993: 159).

La nueva realización del calco nos ha permitido apreciar un mayor número de elementos del tercio delantero del animal, detalles o zonas poco visibles, pudiéndose confirmar la existencia del elemento lineal ya descrito en el primer estudio de conjunto, que interpretamos igualmente como una lanza o bien una flecha de gran longitud clavada en el pecho del toro.

\subsection{Ceja de Piezarrodilla}

El abrigo se abre en la parte alta de la misma formación de arenisca que el conjunto anterior, a unos $100 \mathrm{~m}$ de altura con respecto al fondo del valle y con orientación SE, ocupando una posición estratégica de primer

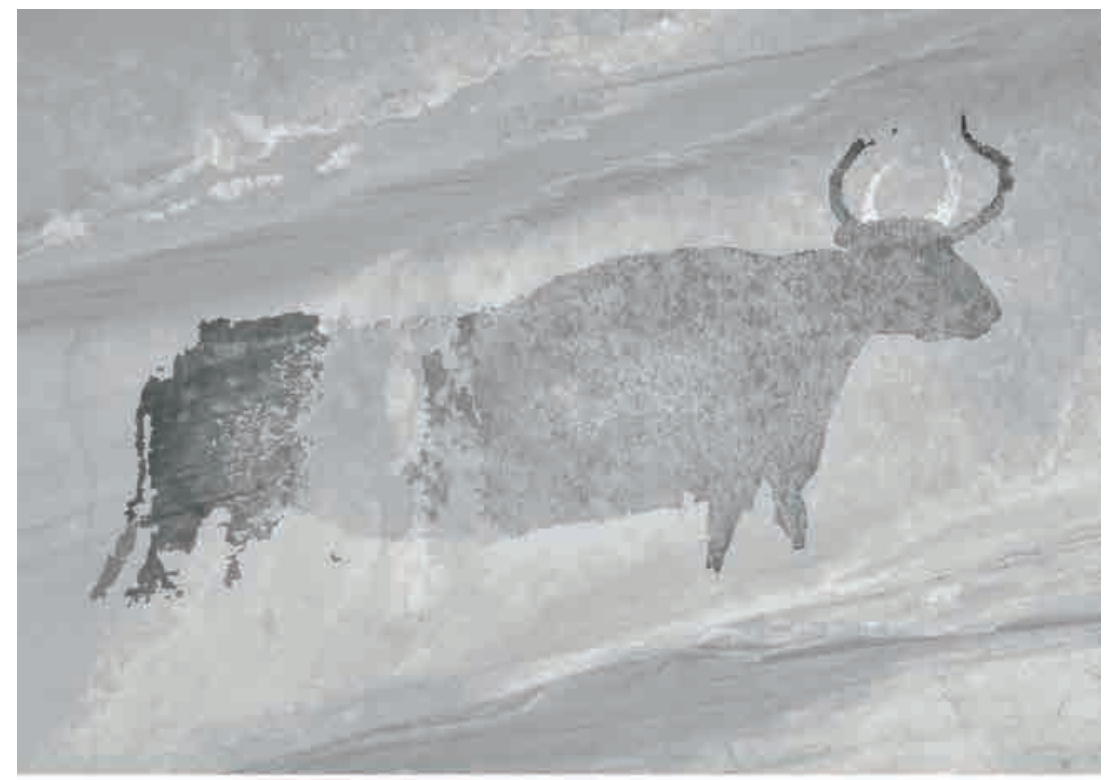
orden para el control visual del entorno.

El conjunto fue descubierto por Breuil en 1926, dándose una noticia preliminar del mismo en un breve artículo publicado por Obermaier (1927) y siendo publicado poco después por ambos investigadores (Obermaier y Breuil, 1927). Más tarde sería mencionado nuevamente en los trabajos de Beltrán (1968: 148 y 1993: 159), aunque sin profundizar en su análisis o realizar nuevos calcos, analítica que se lleva a cabo con los trabajos desarrollados por Piñón $(1981,1982)$.

En 1986, J. Sáez descubrió una segunda representación en el mismo abrigo. Su mal estado de

conservación y escasa visibilidad ha determinado que haya pasado totalmente desapercibida, incluso en trabajos de revisión posteriores a su descubrimiento (Beltrán, 1993; Utrilla, 2000), de manera que con este estudio se da a conocer la nueva representación.

- Motivo 1. Gran toro orientado a la derecha, localizado a $250 \mathrm{~cm}$ del suelo del abrigo y a $108 \mathrm{~cm}$ del suelo sobre una repisa. Este sector del abrigo tan sólo cuenta con $145 \mathrm{~cm}$ de altura máxima. Dimensiones: $66,5 \mathrm{~cm}$ de longitud y $35 \mathrm{~cm}$ de altura. 

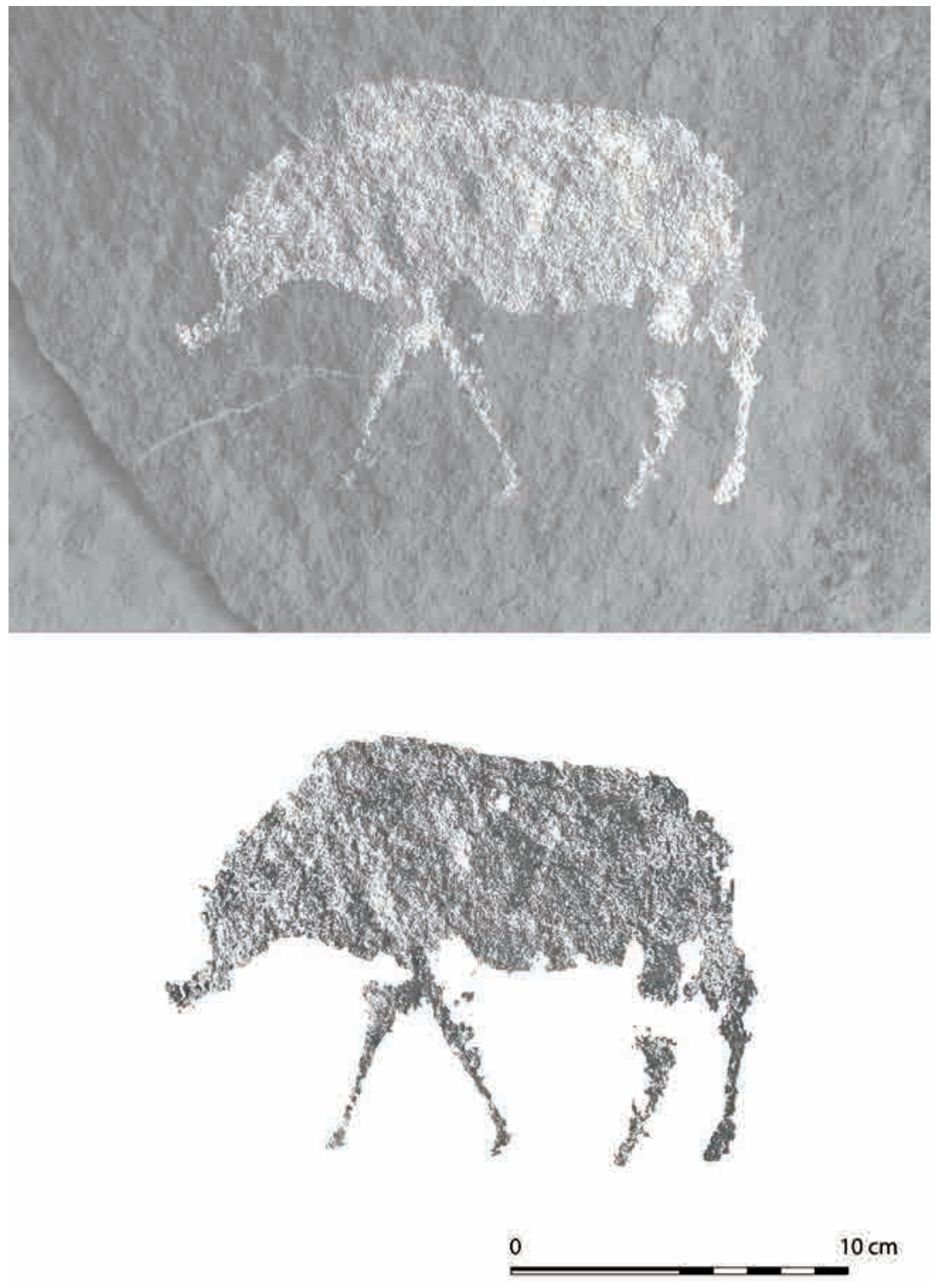

FIG. 4. Calco del motivo 2 de Ceja de Piezarrodilla. animal, en gran medida por la plasmación de una voluminosa giba, un cuello corto y un ancho pecho, amplificado por el escaso desarrollo de las patas. Con todo, en términos globales, la masividad aludida se difumina por la marcada desproporción del cuerpo del animal, excesivamente alargado. Es ésta una de las principales características estilísticas del motivo, junto con las patas cortas.

Merece la pena destacar la diferencia observada entre los dos pares de cuernos conservados, gracias a los cuales es posible argumentar la existencia de un repintado global del motivo.

Así, se aprecian dos cuernos en perspectiva torcida de color blanco en el centro de la cabeza del animal. Se trataría, junto a escasos restos apenas perceptibles en diferentes zonas de la mitad delantera del animal, de lo único conservado del motivo original. Sobre esta figura se realizaría un repintado en color negro que, en esencia, repasaría la forma del zoomorfo previo a excepción de los cuernos, para los que se recurre a una nueva disposición y perspectiva. En esta segunda fase se confeccionarían unas astas mucho más finas y realistas, con forma de lira, aunque todavía en perspectiva frontal.
La representación (Fig. 3) sigue un patrón de proporciones alejado del realismo más puro de las representaciones de otros conjuntos levantinos, mostrando un cuerpo excesivamente alargado con respecto a las patas. Sin embargo, presenta una serie de rasgos de componente naturalista como el detalle de la oreja, la joroba o la cola, larga y empenachada.

Las formas del motivo se presentan, en general, rotundas, sobre todo en la mitad delantera del
- Motivo 2. Zoomorfo (Fig. 4) localizado a $447 \mathrm{~cm}$ a la izquierda del motivo 1, cerca de la entrada del abrigo, tan sólo a $14 \mathrm{~cm}$ de la confluencia formada por dos lienzos de roca que convergen en un ángulo de $110^{\circ}$. Se encuentra a $130 \mathrm{~cm}$ de altura respecto a la repisa y a $241 \mathrm{~cm}$ de altura respecto al suelo del abrigo, en un plano rocoso de orientación diferenciada con respecto a la figura anterior. Dimensiones: $17,5 \mathrm{~cm}$ de longitud x $10 \mathrm{~cm}$ de altura. 
Se trata de un motivo de tonalidad blanca muy mal conservado. No fue percibido en el estudio de Piñón (1982), habiendo pasado prácticamente desapercibido en estudios generales posteriores a su descubrimiento.

El motivo presenta un cuerpo, hasta cierto punto, robusto y caracterizado por la plasmación de unas patas finas y bien modeladas, sobre todo las delanteras, abiertas en ' $v$ ' invertida. El cuello se representó agachado, sin que se haya conservado o completado la cabeza.

La mala conservación general de la representación ha llevado a interpretarla como un posible jabalí (Martínez Utrillas, 2012: 53). Sin embargo, y a partir de la comparación con paralelos existentes en otros conjuntos de la Serranía de Albarracín, consideramos factible su interpretación como un bovino o équido en el que, por factores de conservación, la cabeza se habría perdido o no fue completada.

\section{Comentarios a los conjuntos}

Uno de los aspectos más destacados de los conjuntos analizados es la cuestión del repintado del toro de Ceja de Piezarrodilla. Los primeros estudios aludían ya a la excepcionalidad del repintado en negro de un toro anterior de color blanco, del que se conocería su existencia sólo gracias a que se conservarían intactas las astas en color blanco, asomando entre la cornamenta de color negro de factura posterior.

El análisis realizado por Piñón (1981, 1982) alude a la existencia de tres fases decorativas en lugar de dos. En un primer momento se realizaría la figura en tonos blanquecinos (Piñón, 1981: fig. 1a) cuya existencia aparece remarcada por la cornamenta en medialuna o perspectiva frontal que se observan entre las astas en lira de tonalidad negruzca pertenecientes a la fase más reciente. Para la segunda fase decorativa (Piñón, 1981: fig. 1b) el citado investigador advierte ciertos restos de tonalidad clara, ligeramente anaranjada, en la cabeza, cuernos, pecho y lomo del bóvido que aparecerían debajo del pigmento negro. En esta fase, la cornamenta adoptaría el formato en lira que sería respetado en el siguiente repintado. En un tercer momento (Piñón, 1981: fig. 1c) se repintaría nuevamente toda la figura, en esta ocasión en color negro con las ya mencionadas astas en forma de lira, orejas, cruz bien marcada y cola, todos ellos elementos de componente naturalista que contrastan con el desproporcionado alargamiento del cuerpo y las cortas patas.

Como ya se ha señalado, en la descripción de Piñón se habla de una serie de restos blanquecinos de componente anaranjado (fig. 1b) que aparecerían sepultados por los "empastes negruzcos del repintado" (Piñón, 1981: 10; 1982: 150), observándose sobre todo en la cabeza, pecho, lomo y cuernos. Esta observación contrasta abiertamente con la más tempranamente realizada por Obermaier y Breuil (1927: 531 y lám. XIv), quienes subrayan que el color negro es "más intenso en la cabeza y en los bordes de la silueta", precisamente las zonas en las que Piñón remarca la presencia del repintado en color claro (fig. 1b) y que, según parece, no se apreciaba en el primer estudio del conjunto. La consulta de la documentación fotográfica llevada a cabo en 1972 por M. Almagro (Corpus Digital de Pintura Rupestre Levantina) $)^{3}$ permite, asimismo, constatar tan sólo dos fases decorativas, sin que en ningún momento se pueda apuntar la existencia de la tercera fase aludida por Piñón.

Siguiendo esta línea, en la mayoría de los estudios posteriores al de Piñón se hace mención, exclusivamente, a la existencia de un único repintado o dos fases decorativas: negro sobre blanco (Beltrán, 1986: 45; 2002: 101), apuntando la cuidadosa superposición con la que "aunque cambiase la cronología absoluta no se alteró el estilo” (Beltrán, 1993: 159), si bien en un trabajo de síntesis sobre el arte rupestre en Aragón se indicaba que el bóvido sería "repintado al menos en tres ocasiones" (Utrilla, 2000: 35).

Tras la reciente documentación y análisis realizados en el conjunto, consideramos que se debería pensar en que los restos anaranjados aludidos por Piñón fueran, en realidad, el componente visual de un proceso de degradación progresivo motivado, quizá, por el crecimiento de líquenes o cianobacterias, cuyos primeros procesos de crecimiento se corresponderían con la descripción realizada por Piñón y que, con el paso del tiempo, se habría ido

\footnotetext{
3 La consulta de los archivos fotográficos históricos aparece como un elemento de análisis de importancia para conocer la evolución diacrónica de los conjuntos rupestres (http://www.prehistoria.ceh.csic.es/AAR/frames.php).
} 
generalizando por toda la figura ${ }^{4}$. No se trataría, por tanto, de una fase decorativa diferenciada sino de un proceso degenerativo de la pintura por el afloramiento de líquenes o cianobacterias.

Durante los trabajos de revisión hemos podido observar una marcada diferenciación cromática de la gran figura de toro con respecto a imágenes de archivo previas ${ }^{5}$. Así, tanto en las fotografías aportadas por los estudios de Piñón (1982), como en las realizadas en los años ochenta del s. Xx por parte de un equipo dirigido por Beltrán, se observa claramente un tono negro generalizado en la totalidad de la figura, sobre todo en la mitad delantera.

El tratamiento digital de las fotografías mediante DStretch@ ha resultado ser de gran utilidad no sólo para una mejor lectura del motivo y la elaboración de los calcos digitales, sino también para tratar de aislar la posible existencia de la capa de agentes bióticos y determinar su distribución por la superficie del motivo pintado. Como se puede apreciar, los agentes erosivos se distribuyen sólo por la mitad delantera del animal, exclusivamente sobre la superficie de color negro, ya que las astas de color blanco infrapuestas al repintado negro no parecen haberse visto afectadas (Fig. 5).

Los cuartos traseros del animal todavía conservan la coloración negruzca, sin que resulten evidentes los elementos de tonalidad blanquecina observados en la mitad delantera. La escorrentía que afecta directamente a parte de la mitad trasera del cuerpo del toro, y que ha hecho desvaírse el pigmento, parece determinar el límite de expansión del posible agente biótico que afecta a la parte delantera.

$\mathrm{Al}$ margen de aspectos técnicos y de coloración, los conjuntos analizados presentan dos motivos de tendencia naturalista pero con rasgos estilísticos bien

\footnotetext{
${ }^{4}$ Hoy se ha perdido buena parte de la visibilidad de la pintura, con una tonalidad blanquecina superpuesta al motivo que deberá ser analizada para conocer su origen y el impacto en la conservación del motivo. Esta particularidad fue dada a conocer por nosotros mismos a la Dirección General de Patrimonio Cultural del Gobierno de Aragón, desde donde se han iniciado los trabajos de análisis de laboratorio.

5 Es muy posible que la diferenciación cromática apuntada se deba a que en trabajos previos se humedeciera la pintura para mejorar su visibilidad. En ningún momento en los trabajos recientes que llevamos a cabo sobre los conjuntos revisados se humectaron los motivos pictóricos.
}

diferenciados. La actitud de los animales, emplazamiento y naturaleza de la acción contenida en la escena imprimen igualmente un factor divergente en sus composiciones y realizaciones.

El de Cerrada de Tío Jorge es uno de los escasos ejemplos de bóvido con un venablo clavado en la Sierra de Albarracín. Sólo en Prado del Navazo sería representado otro herido, si bien en este caso el elemento clavado, al ser corto y mostrarse en relación con un arquero, se identifica perfectamente con una flecha y no con un venablo o lanza. Además, la diferenciación cromática entre el toro referido -de color blanco-y de la flecha y arquero -de color negro- podrían apuntar a diferentes fases de ejecución o incluso a una escena de carácter acumulativo (Martínez Bea, 2008).

Resultan evidentes los paralelos cercanos para el toro del abrigo de Ceja de Piezarrodilla (Fig. 6): grandes figuras de toros con cuerpos alargados, patas cortas, cuellos por lo general masivos, actitud predominantemente estática, astas en medialuna y frecuente uso de color blanco, si bien algunos casos aparecen realizados en tonalidades negruzcas. Así, encontramos claros paralelos en conjuntos cercanos, como en el recientemente descubierto de Hoya de Navarejos III (Tormón) o en los muy conocidos abrigos con grandes figuraciones de bóvidos de Albarracín: Prado de las Olivanas, Prado del Navazo, Cocinilla del Obispo y los, bastante menos referidos, del Arquero de los Callejones Cerrados y del Medio Caballo.

Quizá, un elemento que singulariza el toro de la Ceja de Piezarrodilla respecto a otros de la zona de Albarracín con evidentes rasgos estilísticos comunes es, precisamente, que aparece como una representación aislada, ajena a las composiciones en grupo de abrigos como los del Prado del Navazo, Cocinilla del Obispo, Prado de las Olivanas, Medio Caballo o en el de Hoya de Navarejos III, todavía en fase de estudio.

A pesar de su conservación incompleta, se documentan algunos paralelos para el segundo de los motivos del abrigo de Ceja de Piezarrodilla (Fig. 6, n. $\left.{ }^{\text {os }} 8-9\right)$. No resultan extrañas las figuraciones, fundamentalmente de bóvidos, de medianas dimensiones en una actitud y disposición exactas a las del cuadrúpedo que nos ocupa. Así, se documentan en los motivos 11 y 13 de Prado del Navazo, también de color blanco y sin la cabeza 
conservada (Piñón, 1982: 45); o los motivos 14 y 15 del Prado de las Olivanas, ambos de color negro aunque en el primero se llega a apuntar la posible existencia de un repintado de este color sobre una figura previa de tonalidad blanca (Piñón, 1982: 155-156). Idéntica disposición se documenta también en las dos figuraciones en color blanco de Prados de Arejos i (Tormón), aunque en este caso una de las representaciones se corresponde con un caballo.

\section{Conclusiones}

Resulta interesante subrayar la variación estilística de los dos grandes bóvidos de la zona de la Casa Forestal de Tormón. Si bien se aprecian algunos elementos compartidos, lo cierto es que las disconformidades observadas-longitud del cuello, anchura de la cabeza, actitud, coloración, etc.- hacen que podamos hablar de, al menos, tres momentos figurativos diferenciados: uno para cada una de las figuraciones de grandes bóvidos documentadas en cada abrigo y otro más relativo al repintado en negro en la Ceja de Piezarrodilla.

Acerca de este repintado no creemos posible hablar de la existencia de tres fases de repintados para el toro de Ceja de Piezarrodilla, sino tan sólo de dos -negro sobre blanco-, pudiéndose explicar la alusión a la tercera fase de repintado, argumentada por Piñón, a partir de su definición como un posible agente erosivo de naturaleza biótica.

Así pues, además de eflorescencias salinas, buena parte del bóvido estaría seriamente afectada por el crecimiento de algún tipo de agente biótico -liquen, cianobacteria, etc.- que se habría expandido sólo, o fundamentalmente, sobre el pigmento de color negro de la parte delantera de la representación animal, quedando libre o minimizada la afección en la parte trasera. La existencia de una zona erosionada por una escorrentía de agua -que habría eliminado o lavado el pigmento negro- podría haber actuado a modo de 'cortafuegos' ante el avance del posible microorganismo. Estudios
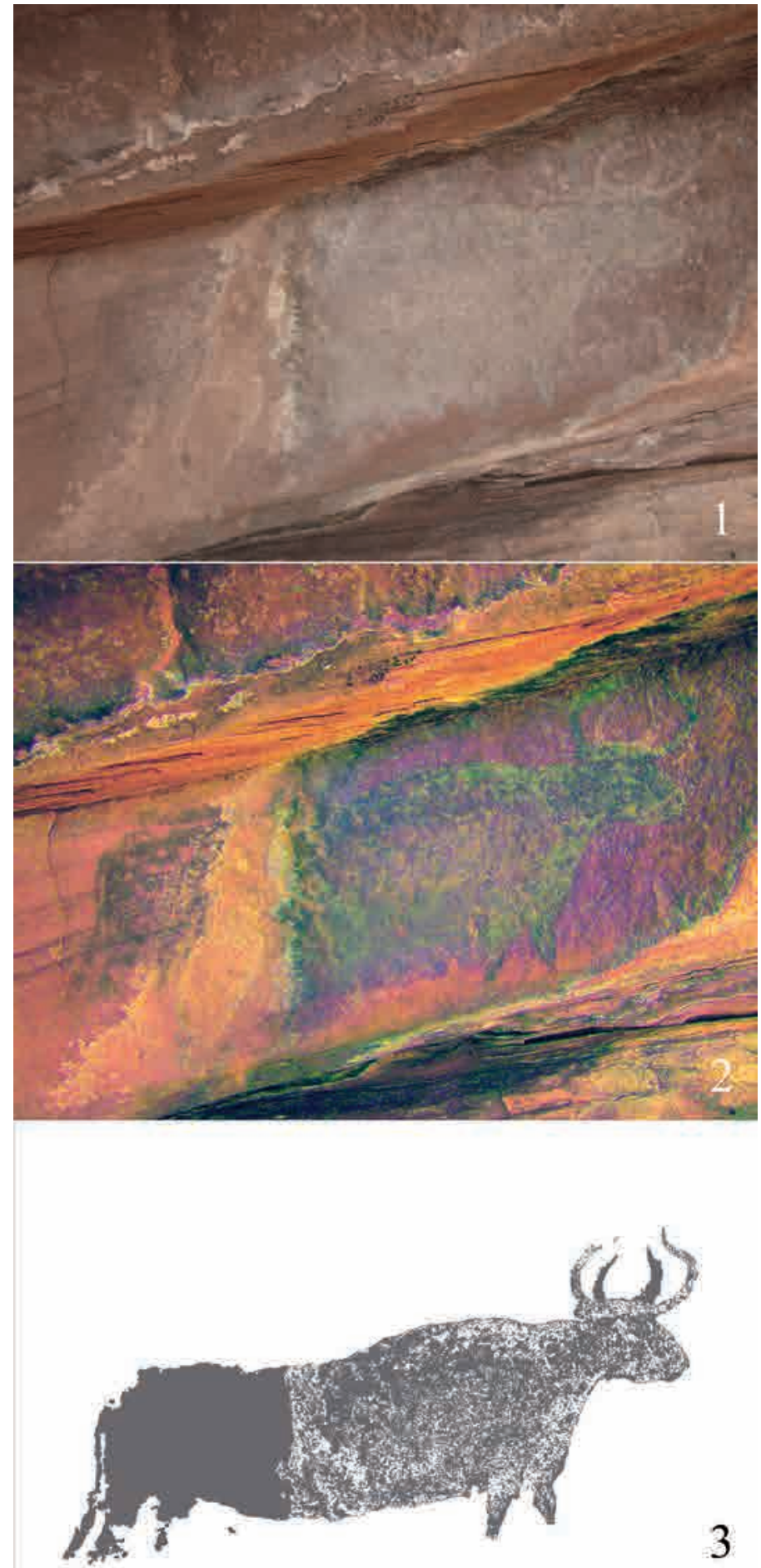

FIG. 5. Bóvido de Ceja de Piezarrodilla: 1) fotografia; 2) imagen retocada en la que se aprecia la posible colonia de cianobacterias sobre el color negro del toro; 3) calco en el que se refleja la superficie afectada por el crecimiento biótico (en color blanco). 

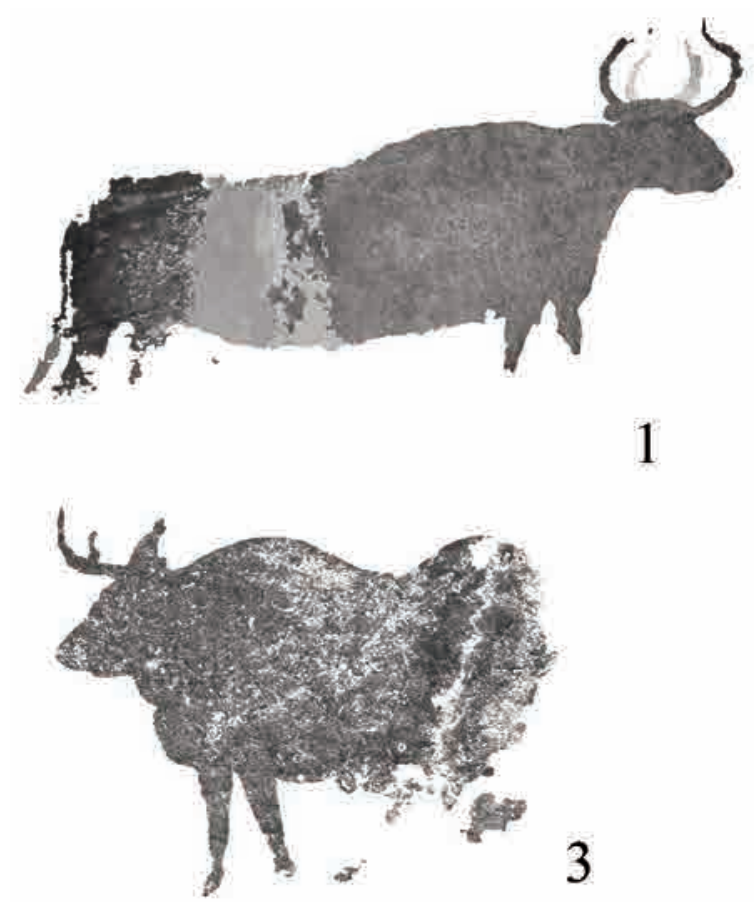

3
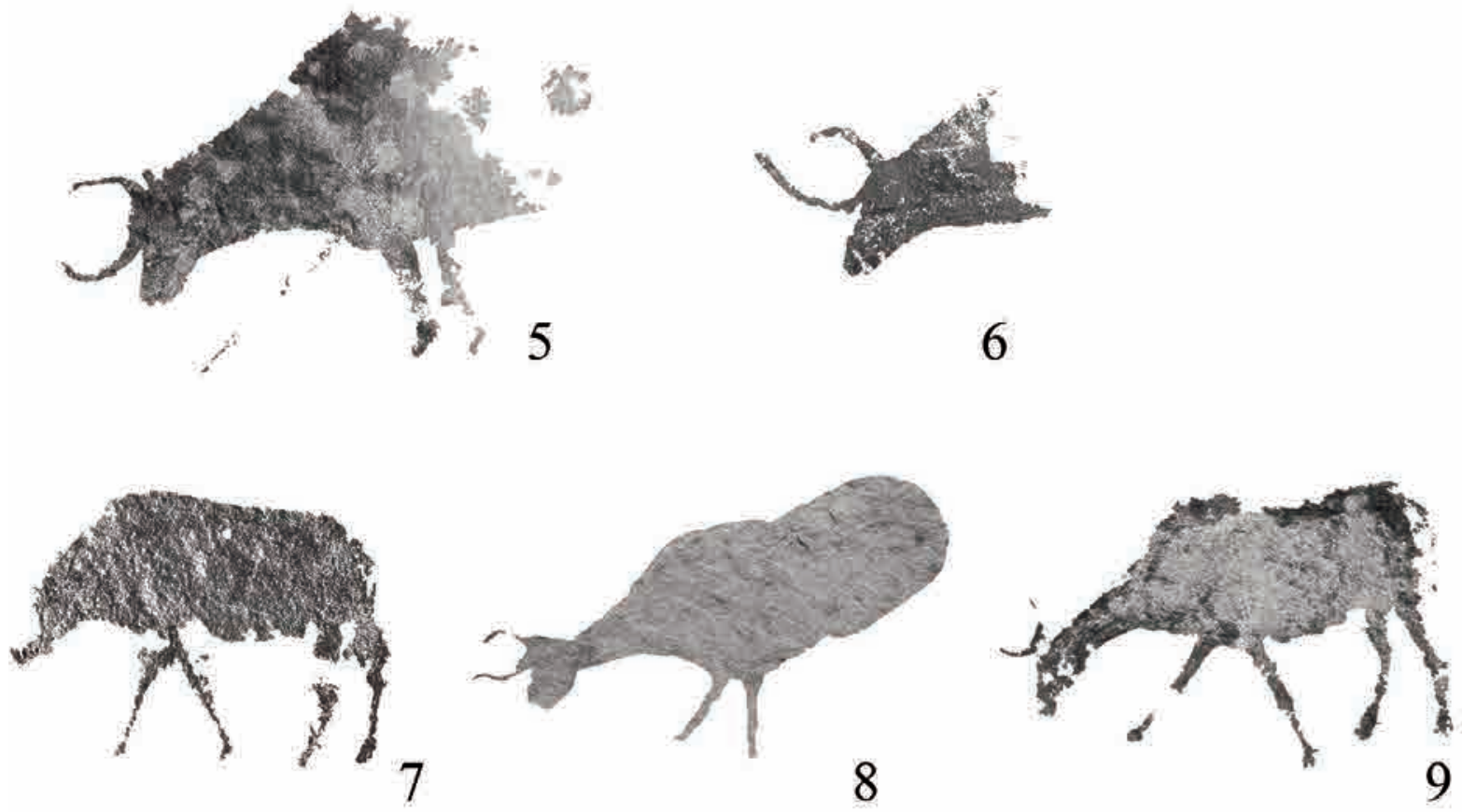

Fig. 6. Paralelos de los motivos estudiados: 1) Ceja de Piezarrodilla; 2) Cocinilla del Obispo; 3) Medio Caballo; 4 y 6) Hoya de Navarejos III; 5) Cerrada del Tío Jorge; 7) Ceja de Piezarrodilla; 8 y 9) Prado de las Olivanas. 
previos en diversos conjuntos rupestres apuntan a un fenómeno de selección de microorganismos que dan lugar a una distribución diferencial entre los alrededores de los abrigos y estos mismos (Alloza et al., 2009: 323) y, como parece ocurrir en el caso que nos ocupa, consideramos que quizá también en un mismo motivo.

El análisis y la definición de la naturaleza de este elemento erosivo se impone como un aspecto básico para tratar de encontrar un tratamiento efectivo y rápido para la conservación de la pintura. Sin duda, el análisis de la composición del pigmento negro, sobre el que parece expandirse exclusivamente la capa blanquecina referida, aportaría datos relevantes.

El conjunto del arte rupestre de la Sierra de Albarracín se define como un núcleo artístico heterogéneo, aspecto perfectamente aplicable a algunos de los temas más singulares de los que contiene, como el de los bóvidos. Éstos ... "tienen poco que ver con los habituales del arte levantino (por color, tamaño, convenciones...)" (Beltrán, 1995: 291), si bien no se debería únicamente poder apuntar a una cronología antigua (Beltrán, 1968; Ripoll, 1968) sino también sugerir, en conjunción con otros aspectos, una atribución cronocultural reciente (Cruz, 2004, 2005; Martínez Bea, 2008) ${ }^{6}$.

Aspectos como los apuntados en las páginas precedentes subrayan la importancia de concretar y matizar la validez de clasificaciones cromáticas en general y para el núcleo de la Sierra de Albarracín en particular. La tradicionalmente aceptada ordenación cromática de las figuras del área analizada: blanco, rojo claro, rojo oscuro y negro, de más antigua a más reciente (Beltrán, 1986: 40), resulta, cuando menos, matizable con la observación de algunos casos en los que no se cumple. Así, la superposición negro sobre blanco observada en el toro de la Ceja de Piezarrodilla encuentra su equivalente inverso -blanco sobre negro- en el Prado del Navazo. En este conjunto, tal y como se ha destacado en otros estudios, un gran toro blanco -motivo 5- se superpone a un trazo en negro definido como una posible flecha disparada por un arquero negro semiesquemático (Martínez Bea,

${ }^{6}$ Cf. también Martínez Bea, M.: Variabilidad estilistica $y$ distribución territorial del arte rupestre levantino en Aragón: el ejemplo de La Vacada (Castellote, Teruel). Tesis doctoral inédita presentada en 2005 en la Univ. de Zaragoza.
2008: 142), al mismo tiempo que el citado bóvido blanco superpone sus patas posteriores sobre los cuartos traseros de un toro de tonalidad negruzca -motivo 9- (Piñón, 1982: 43 y 52).

Los motivos analizados, en conjunción a los de conjuntos de la Serranía de Albarracín, apuntan a la existencia real de una temática de raigambre local: grandes figuraciones de toros que, o bien aislados o en rebaños, subrayan su carácter mayestático. Con todo, es posible advertir que en dicha tradición existen diferencias en rasgos estilísticos que se centran esencialmente en el tratamiento de detalles anatómicos -sobre todo en la morfología de la cabeza y de las patas-. Así, y atendiendo a las grandes representaciones de bóvidos, es posible encontrar tanto algunos ejemplos de carácter marcadamente naturalista, sobre todo en la realización de las cabezas -Ceja de Piezarrodilla, motivo 7 de Cocinilla del Obispo, motivo 4 de Medio Caballo, motivo 6 del Arquero de los Callejones Cerrados- junto a otros con rasgos más estilizados, convencionalismos que les alejan del estricto naturalismo y que algunos autores han definido como "naturalismo tosco" o "naturalismo estilizado" (Piñón, 1982: 189-190). En realidad, a estos dos últimos grupos responderían la mayoría de las grandes figuraciones de bóvidos de la Sierra de Albarracín, casi todas con cuerpos alargados, cuellos y patas cortas o morros alargados, todo lo cual dota a las representaciones de un aspecto, en general, pesado y poco grácil, alejado del aspecto de las figuraciones de toros levantinas de otras zonas geográficas: La Vacada (Martínez Bea, 2009), El Torico (Bea, 2012a), El Cantalar I (Bea y Domingo, 2009), Val del Charco (Beltrán, 2002; Bea, 2012b) en la zona del Bajo Aragón-Maestrazgo turolense o los del Cingle de la Cova Remigia o abrigo viI de Gasulla (Porcar, 1947; Domingo et al., 2003), Mas d'en Josep (Domingo et al., 2003) o Mas Blanc (Domingo et al., 2003) en el Maestrazgo castellonense.

Las grandes representaciones de bóvidos de la Sierra de Albarracín, de las que los ejemplares analizados de Ceja de Piezarrodilla y Cerrada del Tío Jorge forman parte, pueden ser entendidas como una seña de identidad territorial cuya singularidad debe ser aprehendida en el amplio contexto definitorio del arte levantino para tratar de avanzar, precisamente, en una definición más concreta del mismo. 


\section{Bibliografía}

Alloza, R.; Arranz, E.; González, J. M.; Baldellou, V.; Resano, M.; Marzo, P. y Vanhaecke, F. (2009): "La conservación del arte rupestre: estudio de los factores de deterioro y de la composición química de los pigmentos”. En López, J. A.; Martínez, R. y MataMoros, C. (eds.): El arte rupestre del Arco Mediterráneo de la Peninsula Ibérica. 10 años en la lista del Patrimonio Mundial de la UNESCO. Valencia: Generalitat Valenciana, pp. 317-325.

Almagro, M. (1970): Manual de Historia Universal. Madrid.

BEA, M. (2012a): "Nuevas perspectivas de análisis para el arte rupestre del Maestrazgo. Los abrigos del Arquero y del Torico (Castellote, Teruel)", Zephyrus, Lxx, pp. 49-67. http://campus.usal.es/ revistas_trabajo/index. php/0514-7336/issue/view/641.

Bea, M. (2012b): Val del Charco del Agua Amarga. 19132013 Centenario de un descubrimiento. Teruel: Comarca del Bajo Aragón-Dir. General de Bellas Artes y Bienes Culturales.

BeA, M. y Domingo, R. (2009): "Las pinturas levantinas del abrigo de El Cantalar I (Villarluengo-Montoro de Mezquita, Teruel)", Saguntum, 41, pp. 37-46. https:// ojs.uv.es/index.php/saguntum/issue/view/75/showToc.

Beltrán, A. (1968): Arte rupestre levantino. Monografías Arqueológicas, IV. Zaragoza: Univ. de Zaragoza.

Beltrán, A. (1986): El arte rupestre en la provincia de Teruel. Cartillas Turolenses, 5. Teruel: Instituto de Estudios Turolenses.

Beltrán, A. (1993): Arte prehistórico en Aragón. Zaragoza: Ibercaja.

Beltrán, A. (1995): "Sobre el arte levantino, especialmente de Albarracín. Ideas generales para un debate”. En Actas XXI Congreso Nacional de Arqueología (1991, Teruel). Zaragoza: Diput. General de Aragón, pp. 289-315.

Beltrán, A. (2002): Las pinturas rupestres del abrigo de Val del Charco del Agua Amarga de Alcañiz. Zaragoza: Prames.

Collado, O. (1992): Parque Cultural de Albarracín. Zaragoza: Gob. de Aragón.

Cruz Berrocal, M. (2004): "La investigación del arte rupestre desde la Geografía: la pintura neolítica del ámbito mediterráneo de la Península Ibérica”, Trabajos de Prehistoria, 61 (2), pp. 41-62. http://tp.revistas.csic.es/ index.php/tp/issue/view/4.
Cruz Berrocal, M. ${ }^{\text {a }}$ (2005): Paisaje y arte rupestre. Patrones de localización de la pintura levantina. BAR Int. Ser., 1409. Oxford: Archaeopress.

Domingo, I.; López, E.; Villaverde, V.; Guillem, P. y Martínez VAlle, R. (2003): "Las pinturas rupestres del Cingle del Mas d'en Josep (Tírig, Castelló). Consideraciones sobre la territorialización del arte levantino a partir del análisis de las figuras de bóvidos y jabalíes", Saguntum, 35, pp. 9-49. https://ojs.uv.es/index.php/ saguntum/issue/view/135/showToc.

Hernández Pacheco, E. (1959): Prehistoria del Solar Hispano (Orígenes del Arte). Serie de Ciencias Naturales. Madrid: Real Academia de Ciencias Exactas, Físicas y Naturales.

Martínez BeA, M. (2008): “Arte rupestre de Albarracín: la excepcionalidad de un conjunto interior". En Hernández, M. S.; Soler, J. A. y López, J. A. (eds.): Actas del IV Congreso de Neolitico Peninsular. Alicante: MArQ, pp. 141-148.

Martínez BEA, M. (2009): Las pinturas rupestres del abrigo de La Vacada (Castellote, Teruel). Monografías Arqueológicas, 43. Zaragoza: Univ. de Zaragoza.

Martínez Utrillas, L. (coord.) (2012): El Parque Cultural de Albarracin: Arte Rupestre Patrimonio Mundial. Teruel: Parque Cultural de Albarracín.

Obermaier, H. (1927): "Nuevas pinturas rupestres descubiertas en los alrededores de Tormón (Teruel)", Investigación y Progreso, I (1), pp. 1-2.

Obermaier, H. y Breuil, H. (1927): "Las pinturas rupestres de los alrededores de Tormón (Teruel)", Boletín de la Real Academia de Historia, 90, pp. 511-531.

Piñón, F. (1981): "Superposiciones y repintados en la Serranía de Albarracín: elementos para el establecimiento de una cronología relativa". En Altamira Symposium. Madrid, pp. 411-426.

Piñón, F. (1982): Las pinturas rupestres de Albarracin (Teruel). Monografías del Centro de Investigación y Museo de Altamira, 6. Santander: Ministerio de Cultura.

Porcar, J. (1947): "Iconografía rupestre de Gasulla y Valltorta. Representación pictográfica del toro”, Boletín de la Sociedad Castellonense de Cultura, XxiII, pp. 314-324.

Ripoll, E. (1968): "Cuestiones en torno a la cronología del arte rupestre postpaleolítico en la Península Ibérica”. En Simposio Internacional de Arte Rupestre. Barcelona: Diput. Prov. de Barcelona, pp. 165-192.

Utrilla, P. (2000): El arte rupestre en Aragón. Colección CAI 100, n. ${ }^{\circ}$ 56. Zaragoza: Caja de Ahorros de la Inmaculada. 